\title{
COVID-19 and Low Molecular Weight Heparin (LMWH)
}

\author{
Dr. N. S. Neki \\ Professor, Department of Medicine, Govt. Medical College/Guru Nanak Dev Hospital, Amritsar, Punjab, India.
}

IJCRR

Section: Healthcare

Sci. Journal Impact

Factor: 6.1 (2018)

ICV: 90.90 (2018)

\section{INTRODUCTION}

Pandemics always come up with life-threatening issues. An outbreak of "pneumonia of unknown origin" caused by a newly identified virus i.e. a novel coronavirus was witnessed in December 2019 in the Wuhan city of China and is the worst pandemic mankind has seen in the century. ${ }^{1}$ From China, this Covid-19 outbreak has now affected almost every country globally thus crippling every aspect of human life and has been declared as pandemic by WHO; labelling it Global emergency by January 30, 202012

Coronavirus are positive-sense single-stranded RNA viruses (+ ssRNA) and phylogenetically consist of 4 genera i.e. Alpha coronavirus, Beta coronavirus, Gamma coronavirus and Delta coronavirus while Beta coronavirus consists of subgroups A, B , C and D. Epidemics of the severe acute respiratory syndrome coronavirus (SARS-CoV), H5N, Influenza A, H1N1 2009 and Middle East Respiratory Syndrome (MERS-CoV) causing acute lung injury and acute respiratory distress syndrome (ARDS) resulting in increased mortality have occurred in the past ${ }^{3}$

In India, the first case of the Covid 19 pandemic was reported from Kerala on January 302020 . Globally the infection rate is rising and overall reported mortality varies from $4.3 \%$ to $15 \%$. However mortality in critically ill has been reported high from $15 \%$ to $78 \%{ }^{4}$. Elderly patients, children and those with co-morbidities and immunocompromised subjects are more vulnerable to infection . COVID-19 affects each organ/tissue of the body, starting from brain to foot.

There is high risk of cardiovascular related deaths within weeks in Covid 19 patients. Covid 19 infection has been associated with inflammation and prothrombotic state along with rise in fibrin, fibrin degradation products, fibrinogen and D-dimers. Hence treatment with unfractionated and low molecular weight heparin (LMWH) or as prophylaxis carries excellent prognosis in severe covid-19 patients with coagulopathy. LMWHs are a new class of anticoagulants derived from unfractionated heparin (UFH) indicated for the treatment of venous thrombosis, arterial thrombosis, myocardial infarction and prevention of thrombus.

Unlike UFH, these LMWHs have good pharmacokinetic and pharmacodynamic profile and predictable anticoagulant response and they act by inhibiting the final common pathway of the coagulation cascade ${ }^{5,6}$

As an alternative to fondaparinux, low-dose UFH, Vitamin K antagonists or direct oral anticoagulants, LMWH is the treatment of choice for high risk surgical patients especially abdominal or pelvic cancer surgery and orthopaedic surgery because of their better efficacy, safety profile, cost effectiveness and availability of large clinical data. As Covid19 patients with severe illness are exposed to high risk of thromboembolic events, there are great chances of diffuse hyperinflammatory response further being precipitated by pro coagulation induced hypoxia occurring as a result of prolonged immobilization of these patient Ultimately this process leads to cytokine storm and development of thromboembolism. Heparin is anticoagulant and antithrombotic. LMWHs show favorable response in patients with high levels of D-dimer and prolonged coagulation tests. Simultaneously CT pulmonary angiography must be done in patients having high D-dimer levels more than or equal to $500 \mathrm{mg}$ per litre. LMWH is also given in cases of confirmed pulmonary embolism ${ }^{7}$

According to WHO, venous thromboembolism is a major cause of mortality and morbidity in hospitalized covid-19 patients and LMWH is the treatment of choice for VTE prophylaxis.

LMWHs exhibit little effect on PTT and whole blood clotting time and unlike UFH, LMWHs also possess other properties like anti-inflammatory and antiviral thus decreasing coronary thrombi, pulmonary emboli, microvascular ischemia, decreased lung inflammation and improving oxygenation

Examples of LMWH include Enoxaparin, Reviparin, Nadroparin, Adreparin and Dalteparin. In a study involving 449 patients with severe Covid-19, 99 patients having high levels of d dimer were given prophylactic dose of LMWH i.e. enoxaparin $1 \mathrm{mg} / \mathrm{kg}$ body weight for 7 to 10 days twice daily to get an aPTT target of $60-85$ and 28 day mortality was found to be low. Heparin downgrades interleukin-6 which is increased in covid-19 patients. So LMWH is treatment of choice as anticoagulant in high-risk patients of TE. ${ }^{9}$

Heparins carry risk of bleeding which is less common with LMWH and Fondaparinux. Unlike heparin, Fondaparinux may be used for patients with heparin induced thrombocytopenia along with protamine sulphate. Other side effects include deranged LFTs and osteoporosis. UFH may be used in special cases when creatinine clearance is less than $15 \mathrm{ml}$ per minute or renal replacement therapy. Contraindications to all heparins include hemorrhagic disorders, peptic ulcer disease, recent intracerebral hemorrhage, severe hypertension, recent surgery and trauma.

\section{CONCLUSION}

As VTE and DIC are common complications in majority of hospitalized covid-19 patients, so use of LMWH has been recommended in such patients for Thrombus prophylaxis but keeping in view Risk Benefit Analysis. On patient's discharge from the hospital, routine VTE prophylaxis is not recommended except for high risk patients. LMWHs are well tolerated with minimal side effects. Numerous efforts are underway to develop multiple covid-19 vaccines and will be given as and when became available.

\section{REFERENCES}

1. Cummings MJ, BaldwinMR, Abrams D , et al. Epidemiology, Clinical course and Outcomes of Critically ill adults with Covid-19 in New York city: A prospective cohort study. Lancet 2020; 395: 1763-70. Doi :10.1016/S0140-6736(20)31189-27.

2. Sohrabi C, Alsafi Z, O'Neill N, Khan M, Kerman A, Al-Jabir A. World Health Organisation declares global emergency: a review of the 2019 novel coronavirus (COVID-19). Int. J. Surg 2020;76:71-76.

3. Shereen, Adnan M. "Covid 19 infection: Origin, Transmission and Characteristics of Human Coronavirus". Journal of Advanced Research 2020;24:91-98. doi:10.1016/j/jare.2020.03.005.

4. Zhou, YuT, DuR, et al. Clinical course and risk factors for mortality of adults in patients with Covid 19 in Wuhan, China: a retrospective cohort study (published correction appears in Lancet 2020;395:1054-62. doi:10.1016/S0140-6736(20)30566-3.

5. Nutescu EA, Burnett A, Fanikos J, et al. Pharmacology of anticoagulants used in the treatment of venous thromboembolism (published correction appears in J Thromb Thrombolysis 2016;41:15-31. doi:10.1007/S11239015-1314-3.

6. 6. Deykin D. "The Risks and Benefits of Heparin Therapy". The Western Journal of Medicine, U.S. National Library of Medicine, March 1982. www.ncbi.nlm.nih.gov/pmc/articles/PMC1273659/

7. Diagnosis, Prevention and Treatment of Thromboembolic complications in Covid 19. Report of the National Institute for Public Health of the Netherlands.

8. Rattan R, et al. Coagulopathy in Covid 19. Review and Recommendation

9. Atallah B, Mallah SI, et al. Anticoagulation in Covid 19. European Heart Journal. Cardiovascular Pharmacotherapy. 30 Apr. 2020. Accessed from http.11/doi.org/10.1093/ehjcvp/pv99036 